\title{
Chemosensory Coding by Neurons in the Coeloconic Sensilla of the Drosophila Antenna
}

\author{
C. Andrea Yao, ${ }^{*}$ Rickard Ignell, ${ }^{\star}$ and John R. Carlson \\ Department of Molecular, Cellular, and Developmental Biology, Yale University, New Haven, Connecticut 06520-8103
}

Odor coding is based on the diverse sensitivities and response properties of olfactory receptor neurons (ORNs). In the Drosophila antenna, ORNs are housed in three major morphological types of sensilla. Although investigation of the Drosophila olfactory system has been expanding rapidly, the ORNs in one of these types, the coeloconic sensilla, have been essentially unexplored. We define four functional types of coeloconic sensilla through extracellular physiological recordings. Each type contains at least two neurons, with a total of at least seven distinct ORN classes that vary remarkably in their breadth of tuning. Analysis of 315 odorant-ORN combinations reveals how these neurons sample odor space via both excitation and inhibition. We identify a class of neurons that is narrowly tuned to small amines, and we find humidity detectors that define a cellular basis for hygroreception in Drosophila. The temporal dynamics of responses vary widely, enhancing the potential for complexity in the odor code. Molecular and genetic analysis shows that a broadly tuned ORN, antennal coeloconic 3B (ac3B), requires the odor receptor gene Or35a for its response in vivo. The activity of ac3B is not required for the response of the other ORN within that sensillum, ac3A. The functional analysis presented here, revealing a combination of highly specialized neurons and a broadly tuned ORN, along with the ancient origin of coeloconic sensilla, suggests that the specificities of these ORNs may reflect basic needs of an ancestral insect.

Key words: olfaction; Drosophila; antenna; coeloconic; physiology; receptor

\section{Introduction}

The first step in odor coding is the differential activation of olfactory receptor neurons (ORNs). Signals generated by ORNs undergo processing in the CNS, but the representation of an odor stimulus - its identity, intensity, and temporal structure-originates in the ORNs, whose diverse sensitivities and complex response properties provide the foundation of odor coding (Hildebrand and Shepherd, 1997).

The primary olfactory organ of Drosophila is the third antennal segment (Fig. 1A), whose surface is covered by sensilla ( $n \sim$ 419 in males and $\sim 457$ in females) (Shanbhag et al., 1999). These sensilla are innervated by up to four ORNs and fall into three major morphological types, the basiconic, the trichoid, and the coeloconic sensilla (Fig. $1 B$ ), as well as one minor type, the intermediate sensilla. The coeloconic sensilla $(n=57$ in males and 54 in females) are the smallest of the types, $5 \mu \mathrm{m}$ in height, and are architecturally distinct (Fig. 1C). Unlike the other types, they contain two walls, an outer and an inner wall, at the base of the

\footnotetext{
Received June 14, 2005; revised July 22, 2005; accepted July 26, 2005.

This work was supported by a National Science Foundation predoctoral fellowship to C.A.Y., by the Fulbright Scholar Program and a postdoctoral grant from the Wenner-Gren Foundations to R.I., and by National Institutes of Health Grants DC-02174 and DC-04729 and a McKnight Investigator Award to J.R.C. We thank W. van der Goes van Naters and E. Hallem for discussion and comments on this manuscript.

${ }^{*}$ C.A.Y. and R.I. contributed equally to this work.

Correspondence should be addressed to Dr. John R. Carlson, Department of Molecular, Cellular, and Developmental Biology, Yale University, P.0. Box 208103, New Haven, CT 06520-8103. E-mail: john.carlson@yale.edu.

R. Ignell's present address: Division of Chemical Ecology, Department of Crop Science, Swedish University of Agricultural Sciences, P.0. Box 44, SE-23053 Alnarp, Sweden.

DOI:10.1523/JNEUROSCI.2432-05.2005

Copyright $\odot 2005$ Society for Neuroscience $\quad$ 0270-6474/05/258359-09\$15.00/0
}

sensillum (Shanbhag et al., 1999). The inner wall encloses the ORN dendrites. The distal half of the sensillum appears longitudinally grooved. It is formed by $\sim 8$ closely apposed cuticular fingers, with grooves between the fingers that allow odors to pass through. Sensilla with this type of morphology have been conserved through hundreds of millions of years of insect evolution. They can be found in many and perhaps all insect orders (Steinbrecht, 1997), suggesting a critical function in chemosensory coding.

Although the basiconic sensilla of Drosophila have been extensively investigated (de Bruyne et al., 1999, 2001; Elmore et al., 2003), its coeloconic sensilla remain essentially unexamined. Preliminary investigation confirmed that at least some of its sensilla respond to odors (Clyne et al., 1997; Park et al., 2002); however, the number, specificities, and response properties of coeloconic ORN classes have not been defined, and their role in odor coding is unknown.

This paucity of information derives primarily from the technical difficulty of obtaining high-quality electrophysiological recordings from coeloconic sensilla. In addition to their small size, they are more susceptible to damage by the electrode and yield recordings with lower signal-to-noise ratios than the other types of sensilla. However, efforts to understand the integrated function of the Drosophila olfactory system (Hallem and Carlson, 2004) require an understanding of each of the three major subsets of olfactory input.

Here, we present a systematic investigation of this major type of olfactory sensillum and its contributions to odor coding. We define four functional types of coeloconic sensillum and seven classes of ORNs. The analysis identifies ORNs that represent ex- 
tremes in breadth of tuning, and recordings reveal diverse patterns of temporal dynamics. We identify humidity receptors and amine detectors. Genetic and molecular analysis reveals that $\operatorname{Or} 35 a$, a member of the Or family of odor receptor genes, is necessary for the function of one ORN class, antennal coeloconic 3B (ac3B). Together, the data suggest a model in which the specificities of coeloconic ORNs reflect basic needs of an ancestral insect.

\section{Materials and Methods}

Drosophila stocks and transgenes. The Or35aGAL4 construct was created using primers to amplify a $5.0 \mathrm{~kb}$ DNA sequence immediately upstream of the translation initiation codon of Or $35 a$ and $1.2 \mathrm{~kb}$ of sequence downstream of the stop codon; the $5^{\prime}$ end of the downstream region was $16 \mathrm{bp}$ upstream of the Or35a stop
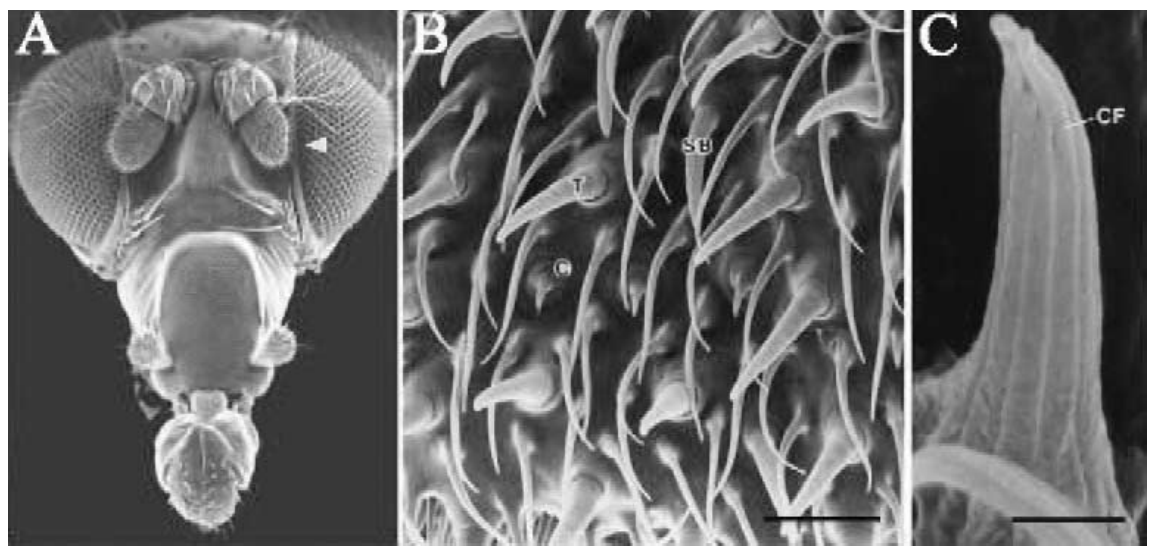

Figure 1. Coeloconic sensilla. $\boldsymbol{A}$, Head of Drosophila. The arrowhead indicates the third antennal segment. $\boldsymbol{B}$, Surface of the antenna, showing the coeloconic sensillum (C), small basiconic sensillum (SB), and trichoid sensillum (T). Scale bar, $10 \mu \mathrm{m}$. C, Coeloconic sensillum. CF, Cuticular fingers. Scale bar, $0.5 \mu \mathrm{m} . \boldsymbol{B}$ and $\boldsymbol{C}$ are from Shanbhag et al. (1999).

codon. The transgenic construct was injected into $w^{1118}$ flies. For cell-ablation experiments, the Or35a-GAL4 line was crossed to UAS-rpr flies. UAS-rpr/CyO was a gift from B. Hay (California Institute of Technology, Pasadena, CA). The Or35a insertion line, $\mathrm{PBac}\{\mathrm{WH}\} \mathrm{Or} 35 \mathrm{a}^{\mathrm{f} 02057}$ was from the Drosophila Stock Center (Bloomington, IN).

Electrophysiology. The preparation for extracellular single-unit recordings is essentially as described previously (Dobritsa et al., 2003). Canton-S flies aged $<1$ week were used for wild-type recordings. Or 35aGAL4;UAS-rpr flies were allowed to age for 7-10 d before recordings were taken, to allow time for cell death to occur. Electrical activity of the neurons was recorded extracellularly by placing an electrode filled with sensillum lymph ringer (Kaissling and Thorson, 1980) in the base of the sensillum. A reference electrode filled with the same ringer was placed in the eye. Signals were amplified using a patch-clamp amplifier (model 2400; A-M Systems, Carlsborg, WA) in voltage-clamp mode and fed into a computer via a 16-bit analog/digital converter (Digidata 1322A; Molecular Devices, Union City, CA). Impulses during the $0.5 \mathrm{~s}$ period before stimulation and the $0.5 \mathrm{~s}$ during stimulation were counted off-line using the AxoScope software (Molecular Devices). Responses of individual neurons were calculated as the increase (or decrease) in impulse rate (spikes per second) relative to the prestimulus rate. Each sensillum was tested with multiple odorants, and no more than three sensilla were analyzed per fly. The data shown are based on an analysis of 130 different sensilla. Neurons were often damaged during recording, as evidenced by the sudden initiation of high-frequency spike trains, presumably from rapid depolarization after membrane rupture. When a neuron in a sensillum was damaged, we discontinued recording from that sensillum.

Recordings were made by measuring currents in voltage-clamp mode (Redkozubov, 1995; Minor and Kaissling, 2003), which yielded a higher signal-to-noise ratio than we obtained when measuring voltages with a high-input impedance differential amplifier. To confirm that the measurement of currents did not confound comparisons with previous analyses based on measurement of voltages (Clyne et al., 1997; de Bruyne et al., 2001), we performed a direct comparison of odorant responses as determined by measurement of voltages versus currents: we tested the magnitudes and temporal dynamics of odor responses of the ab2A neuron (de Bruyne et al., 2001) and found no differences. Specifically, a 500 ms pulse of 2,3-butanedione produced $176 \pm 13$ spikes/s when measuring voltages and $188 \pm 21$ spikes/s when measuring currents $(n=10$ in each case). A $500 \mathrm{~ms}$ pulse of ethyl acetate produced $262 \pm 6$ and $276 \pm 13$ spikes/s, respectively $(n=10)$. Moreover, a direct comparison of ethyl acetate responses at each $100 \mathrm{~ms}$ time point after odorant delivery up to $2 \mathrm{~s}$ after stimulus revealed no differences in the firing rate at any time $(n=10)$.

Odorant stimuli were prepared in Pasteur pipettes as described previously (Dobritsa et al., 2003). Odorants were from Sigma (St. Louis, MO) and were of the highest grade available (97-99\%, except that phenylacetaldehyde was $>90 \%$ pure). Alcohols are $\mathrm{C} 1$, and ketones are $\mathrm{C} 2$. Chem-
A

ac3

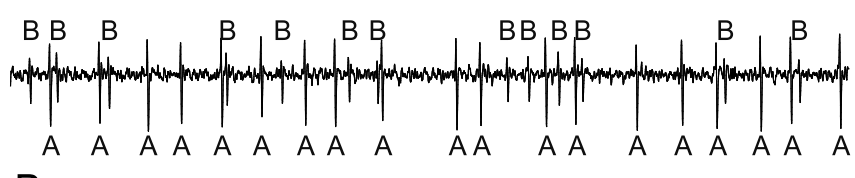

B

ac3

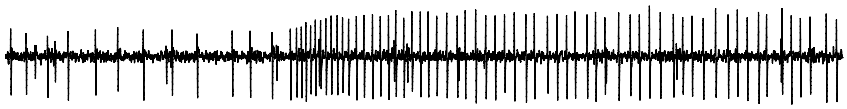

C

isovaleric acid

ac3

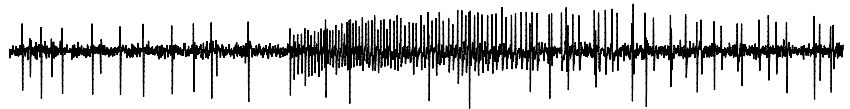

1,4-diaminobutane

ac2

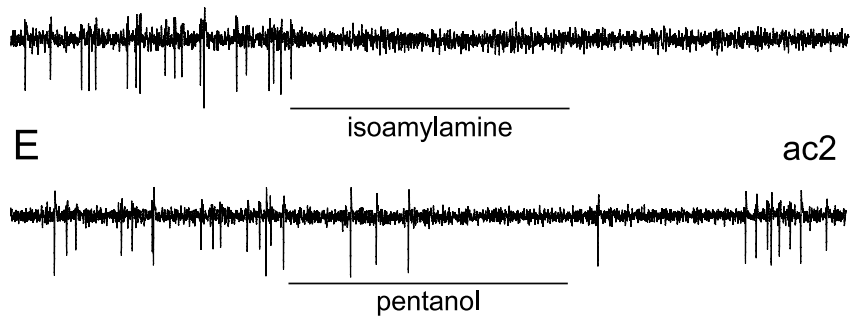

Figure 2. Recordings from the coeloconic sensilla. $\boldsymbol{A}$, Spontaneous activity $(750 \mathrm{~ms})$ of two ORNs in a sensillum later classified as ac3. Spikes of large and small amplitude are designated $A$ and $B$, respectively. $\boldsymbol{B}$, Excitatory response of an $A$ neuron, later classified as ac $3 A$, to isovaleric acid. $\boldsymbol{C}$, Excitatory response of a $B$ neuron, later classified as ac3B, to 1,4-diaminobutane. $\boldsymbol{D}$, Inhibitory response to isoamylamine. Both ORNs are inhibited by the odor. This sensillum was later classified as ac2.E, Inhibitory response of the B cell to pentanol. The A neuron is unaffected. The sensillum was later classified as ac2. Bars represent $0.5 \mathrm{~s}$ pulses of the indicated odors.

icals are racemic mixtures, unless otherwise stated. Propionic acid, butyric acid, ammonia, dimethylamine, 1,4-diaminobutane, and isoamylamine were diluted $10^{-2}$ in $\mathrm{H}_{2} \mathrm{O}$, unless otherwise noted. All other odorants were diluted $10^{-2}$ in paraffin oil (Fluka, Buchs, Switzerland). Stimuli were presented by placing the tip of the pipette through a hole in a glass tube carrying a humidified air stream $(500 \mathrm{ml} / \mathrm{min}) \mathrm{di}-$ rected at the fly and by administering a $0.5 \mathrm{~s}$ pulse of air $(300 \mathrm{ml} / \mathrm{min}$, giving a combined airflow of $800 \mathrm{ml} / \mathrm{min}$ during odor stimulation) through the pipette containing the odorant. All stimuli were used for a maximum of three presentations. The dilution used in most previous 

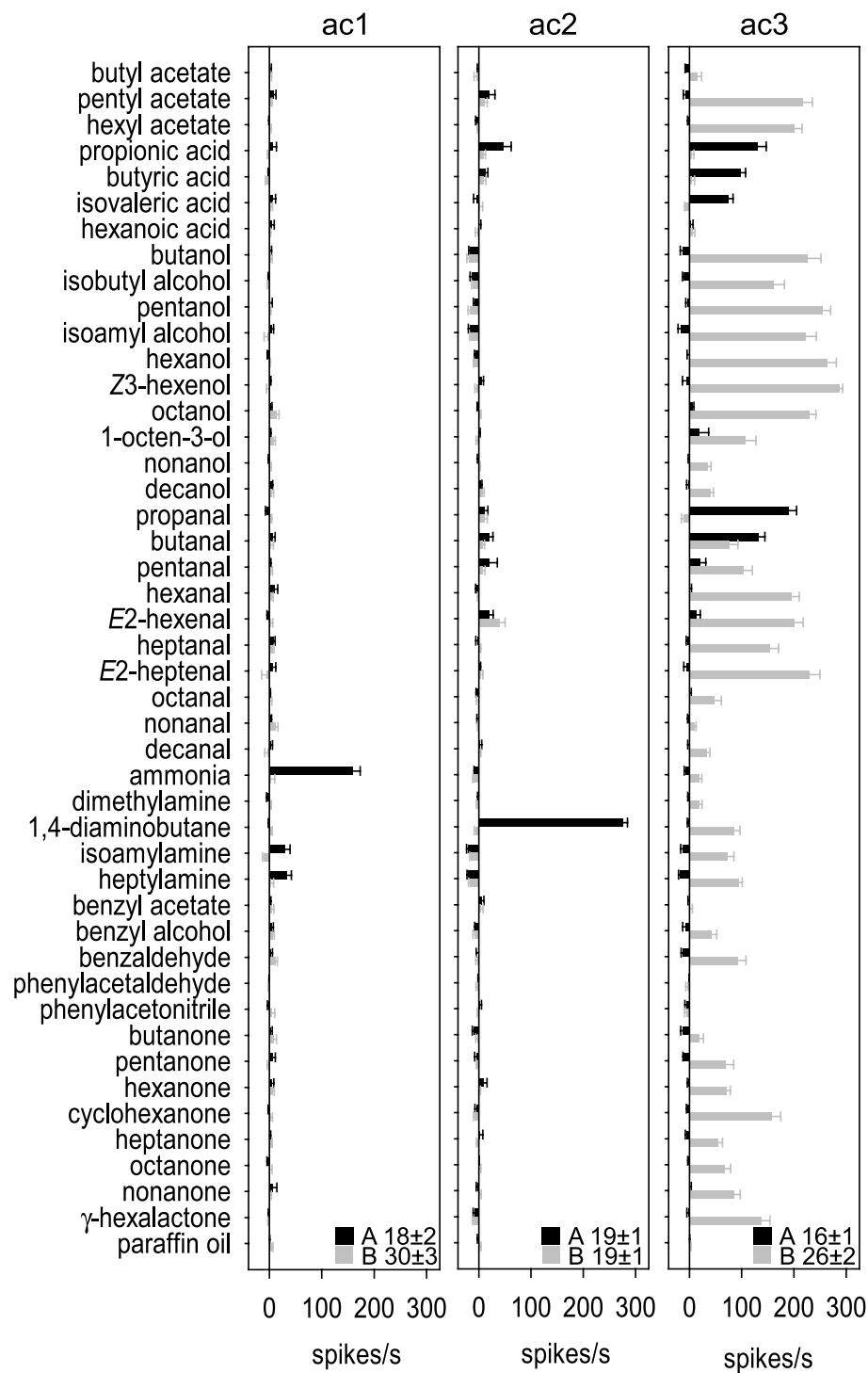

Figure 3. Responses of coeloconic ORNs to a panel of 45 odors. The spontaneous firing frequencies for each ORN are shown in the bottom right corner of each panel. We summed the activities of all ORNs in ac4, because we could not resolve the spikes with confidence. Recordings ( $6 \leq n \leq 40$ ) were each from a different sensillum. Responses to the paraffin oil diluent ranged from $4.4 \pm 2.2$ spikes $/ \mathrm{s}$ (ac2B) to $-1.5 \pm 1.2$ spikes $/ \mathrm{s}$ (ac2A). Error bars indicate SEMs.

characterizations of Drosophila ORNs and odor receptors is $10^{-2}$ (de Bruyne et al., 1999, 2001; Hallem et al., 2004; Goldman et al., 2005; Kreher et al., 2005). Although we refer to the dose in terms of the dilution of the odorant in solvent, i.e., $10^{-2}$, we note that the $0.5 \mathrm{~s}$ pulse of air is further diluted in the humidified air stream, and we do not know how many molecules of odorant are carried from the solvent to the antenna or how this exposure compares with that of a fly in close contact with a fermenting fruit (Hallem et al., 2004).

For humidity recordings, the air stream was switched by a solenoid valve between 0 and $\sim 100 \%$ humidity. The flow rate was kept at a constant $2 \mathrm{~L} / \mathrm{min}$. For $0 \%$ humidity air, we used an ultradry air source (Airgas, Cheshire, CT). To generate the $\sim 100 \%$ humidity air, we bubbled the air through a container of water. A hygrometer (VWR International, West Chester, PA) was used to measure the relative humidities.

\section{Results}

Four functional types of coeloconic sensilla

Extracellular physiological recordings from coeloconic sensilla revealed the spontaneous activities of ORNs. In the case of many

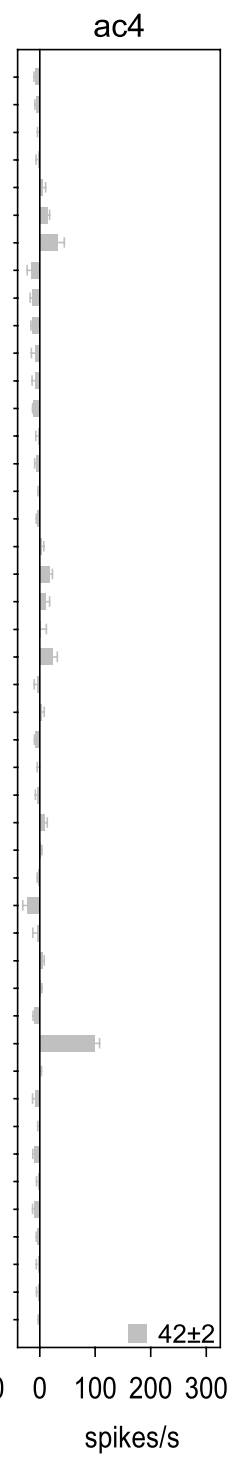

sensilla, two ORNs could easily be classified by virtue of their distinguishable amplitudes: one ORN, termed the A cell, produced spikes of larger amplitude than the other ORN, termed the B cell (Fig. 2A).

Neurons in coeloconic sensilla showed excitatory responses to odors. For example, the A cell shown in Figure $2 B$ produced a train of spikes when stimulated with the odor of isovaleric acid. Different neurons responded to different odors: for example the B cell in the same sensillum responded to the odor of 1,4diaminobutane (Fig. 2C). Although the spike amplitudes shown in Figure 2 remained constant during the course of the recordings, the spike amplitudes in other recordings often changed during periods of intense activation; such changes have been observed previously in recordings of other ORNs from Drosophila (de Bruyne et al., 1999, 2001) and other insect species (Guillet and Bernard, 1972; Rumbo, 1989) and in general do not impair our ability to classify spikes.

In many cases, we observed inhibitory responses to odors: after odor stimulation, the frequency of firing fell below that of the spontaneous frequency. In some instances, both ORNs in a sensillum were inhibited such that little, if any, activity was observed after the odor stimulus (Fig. $2 D)$. In other cases, only one of the ORNs showed strong inhibition (Fig. 2E, B cell).

We chose a set of 45 odors with which to characterize the ORNs of coeloconic sensilla. These odors were chosen to represent different chemical classes, such as acetate esters, organic acids, alcohols, aldehydes, amines, aromatics, and ketones. A number of these odors have been shown previously to play roles in the chemical ecology of Drosophila or other flies, and many are present in fruits (Stensmyr et al., 2003; TNO, 2004).

Extensive recordings, using these odors as stimuli, revealed four functionally distinct types of coeloconic sensilla, which we term ac1, ac2, ac3, and ac4. The magnitudes of the responses of the ORNs in these sensilla to the entire panel of odors are shown in Figure 3 and Table 1. The acl sensillum contains two ORNs. One responded strongly to ammonia, which is an important cue for a number of insects that seek human and animal hosts, including mosquitoes and ticks (Haggart and Davis, 1980; Braks et al., 2001). This ORN showed weaker responses to two other amines, isoamylamine and heptylamine, but none of the other tested compounds. The neighboring ORN showed no responses to any of the compounds. We will refer to the ammonia-sensitive cell as ac1 A and to the nonresponsive cell as ac1B, although we note that acl is an example of a sensillum type in which the ORN classes are difficult to resolve (supplemental Fig. 1, available at www.jneurosci.org as supplemental material). Difficulty in sorting spikes has also been reported for grooved, double-walled sensilla in Anopheles mosquitoes, al- 
though spikes from trichoid sensilla of Anopheles could be classified (van den Broek and Den Otter, 2000).

The ac2 sensillum contains two ORNs, of which ac2A responds strongly to 1,4diaminobutane. We observed only weak excitatory responses, and a few inhibitory responses, to other odors (Table 1, Fig. 3). The other ORN, ac2B, showed only one, weak excitatory response to odors of this set, and a few inhibitory responses.

Our analysis of the ac3 sensillum type defined two ORNs. ac3A is highly sensitive to propanal, and strong excitation was also observed in response to butanal and propionic acid, which are structurally related to propanal. ac3B differs from all other ORNs in this study in its breadth of tuning: it responds to a remarkably high fraction of the tested odors. Of the 45 odors tested, excitatory responses were observed to $36(80 \%)$ (Table 1$)$, and particularly strong responses to most alcohols were measured.

The ORNs in ac4 sensilla gave few excitatory responses, none of which exceeded 100 spikes/s. We were unable to sort the spikes with confidence in our recordings from ac4 and hence have presented the data in Figure 3 and Table 1 as the summation of all spikes from this sensillum elicited by each stimulus (for an example of an ac4 recording, see supplemental Fig. 1, available at www.jneurosci.org as supplemental material). However, it is clear that ac4 contains at least two and perhaps three ORNs, because we could often identify the firing of multiple neurons by the presence of summation events, i.e., overlapping spikes that are recognizable by their altered amplitudes or abnormal shapes; these superimposed spikes must arise from distinct neurons because the time interval between two such spikes is too short to have arisen from a single neuron.

\section{Diverse patterns of temporal dynamics}

The odor responses recorded from coeloconic ORNs revealed diverse patterns of temporal dynamics. In one pattern, the firing frequency rises quickly to a high level and then falls to a lower level, at which it continues firing for a sustained period of time (Fig. 4A). Although the graph in Figure $4 \mathrm{~A}$ shows data only for the first $2.5 \mathrm{~s}$, at the end of which the firing

frequency was $28.7 \pm 5.3$ spikes/s above the spontaneous rate, the elevated firing rate persisted: at $10 \mathrm{~s}$, the frequency was $30 \pm 4.6$ spike/s ( $n=6$; data not shown). A second pattern of temporal dynamics is also marked by a rapid rise, followed by a decline; however, in this case, the firing frequency decays to the spontaneous level by the end of the $2.5 \mathrm{~s}$ recording period (Fig. $4 B$ ). A
Table 1. Response spectra of ORN classes

\begin{tabular}{|c|c|c|c|c|c|c|c|}
\hline \multirow[b]{2}{*}{ Odorant } & \multicolumn{2}{|l|}{$\mathrm{ac1}$} & \multicolumn{2}{|l|}{$\mathrm{ac2}$} & \multicolumn{2}{|l|}{$\mathrm{ac3}$} & \multirow{2}{*}{$\frac{\mathrm{ac} 4}{\Sigma}$} \\
\hline & A & B & A & B & A & B & \\
\hline Butyl acetate & 0 & 0 & 0 & 0 & 0 & + & 0 \\
\hline Pentyl acetate & 0 & 0 & + & 0 & - & ++++ & 0 \\
\hline Hexyl acetate & 0 & 0 & 0 & 0 & 0 & ++++ & 0 \\
\hline Propionic acid & 0 & 0 & ++ & 0 & +++ & 0 & 0 \\
\hline Butyric acid & 0 & 0 & 0 & 0 & ++ & 0 & 0 \\
\hline Isovaleric acid & 0 & 0 & 0 & 0 & ++ & 0 & 0 \\
\hline Hexanoic acid & 0 & 0 & 0 & 0 & 0 & 0 & + \\
\hline Butanol & 0 & 0 & - & - & - & ++++ & 0 \\
\hline Isobutyl alcohol & 0 & 0 & - & - & - & +++ & 0 \\
\hline Pentanol & 0 & 0 & 0 & - & 0 & ++++ & 0 \\
\hline Isoamyl alcohol & 0 & 0 & - & - & - & ++++ & 0 \\
\hline Hexanol & 0 & 0 & 0 & 0 & 0 & ++++ & 0 \\
\hline Z3-hexenol & 0 & 0 & 0 & 0 & 0 & ++++ & 0 \\
\hline Octanol & 0 & 0 & 0 & 0 & 0 & ++++ & 0 \\
\hline 1-0cten-3-ol & 0 & 0 & 0 & 0 & + & ++ & 0 \\
\hline Nonanol & 0 & 0 & 0 & 0 & 0 & + & 0 \\
\hline Decanol & 0 & 0 & 0 & 0 & 0 & + & 0 \\
\hline Propanal & 0 & 0 & 0 & 0 & ++++ & 0 & 0 \\
\hline Butanal & 0 & 0 & + & 0 & +++ & ++ & + \\
\hline Pentanal & 0 & 0 & + & 0 & + & ++ & 0 \\
\hline Hexanal & 0 & 0 & 0 & 0 & 0 & ++++ & 0 \\
\hline E2-hexenal & 0 & 0 & + & + & 0 & ++++ & + \\
\hline Heptanal & 0 & 0 & 0 & 0 & 0 & +++ & 0 \\
\hline E2-heptenal & 0 & 0 & 0 & 0 & 0 & ++++ & 0 \\
\hline 0ctanal & 0 & 0 & 0 & 0 & 0 & ++ & 0 \\
\hline Nonanal & 0 & 0 & 0 & 0 & 0 & 0 & 0 \\
\hline Decanal & 0 & 0 & 0 & 0 & 0 & + & 0 \\
\hline Ammonia & +++ & 0 & 0 & 0 & 0 & + & 0 \\
\hline Dimethylamine & 0 & 0 & 0 & 0 & 0 & + & 0 \\
\hline 1,4-Diaminobutane & 0 & 0 & ++++ & 0 & 0 & ++ & 0 \\
\hline Isoamylamine & + & 0 & - & - & - & ++ & 0 \\
\hline Heptylamine & + & 0 & - & - & - & ++ & 0 \\
\hline Benzyl acetate & 0 & 0 & 0 & 0 & 0 & 0 & 0 \\
\hline Benzyl alcohol & 0 & 0 & 0 & 0 & 0 & + & 0 \\
\hline Benzaldehyde & 0 & 0 & 0 & 0 & - & ++ & 0 \\
\hline Phenylacetaldehyde & 0 & 0 & 0 & 0 & 0 & 0 & ++ \\
\hline Phenylacetonitrile & 0 & 0 & 0 & 0 & 0 & 0 & 0 \\
\hline Butanone & 0 & 0 & - & 0 & - & + & 0 \\
\hline Pentanone & 0 & 0 & 0 & 0 & - & ++ & 0 \\
\hline Hexanone & 0 & 0 & 0 & 0 & 0 & ++ & 0 \\
\hline Cyclohexanone & 0 & 0 & 0 & 0 & 0 & +++ & 0 \\
\hline Heptanone & 0 & 0 & 0 & 0 & 0 & ++ & 0 \\
\hline Octanone & 0 & 0 & 0 & 0 & 0 & ++ & 0 \\
\hline Nonanone & 0 & 0 & 0 & 0 & 0 & ++ & 0 \\
\hline$\gamma$-Hexalactone & 0 & 0 & 0 & 0 & 0 & +++ & 0 \\
\hline Paraffin oil & 0 & 0 & 0 & 0 & 0 & 0 & 0 \\
\hline
\end{tabular}

Responses are indicated as excitatory if the firing frequency exceeds by 15 spikes/s that of the spontaneous frequency. The response exceeded that of the spontaneous frequency as follows: + , by $15 \leq n<45$ spikes $/$ s; ++ , by $45 \leq n<113$ spikes $/$; $;+++$, by $113 \leq n<180$ spikes $/$ s; ++++ , by $180 \leq n$ spikes/s (de Bruyne et al., 2001). The - symbol indicates inhibition to a level $\leq 50 \%$ of the spontaneous firing frequency. $\sum$ indicates that the responses represent the sum of the responses of all ORNs in the sensillum after odor stimulation, because the spikes of the ac 4 ORNs could not be distinguished with confidence. All odorants were diluted $10^{-2}$ in paraffin oil, except for those in italics, which were diluted $10^{-2}$ in water (for explanation of dosages, see Materials and Methods). The 322 entries are each based on recordings from $6 \leq n \leq 40$ different sensilla from $n \geq 4$ different animals. A, A cell; B, B cell.

third pattern (Fig. $4 C$ ) is also characterized by a rapid rise in firing frequency but exhibits a subsequent period of poststimulus quiescence that begins almost immediately after the end of the stimulus. The inhibition is phasic, with the firing frequency returning to its basal level at $1.5 \mathrm{~s}$ in the graph. The final pattern, shown in Figure $4 D$, is one of inhibition, which begins immediately after 

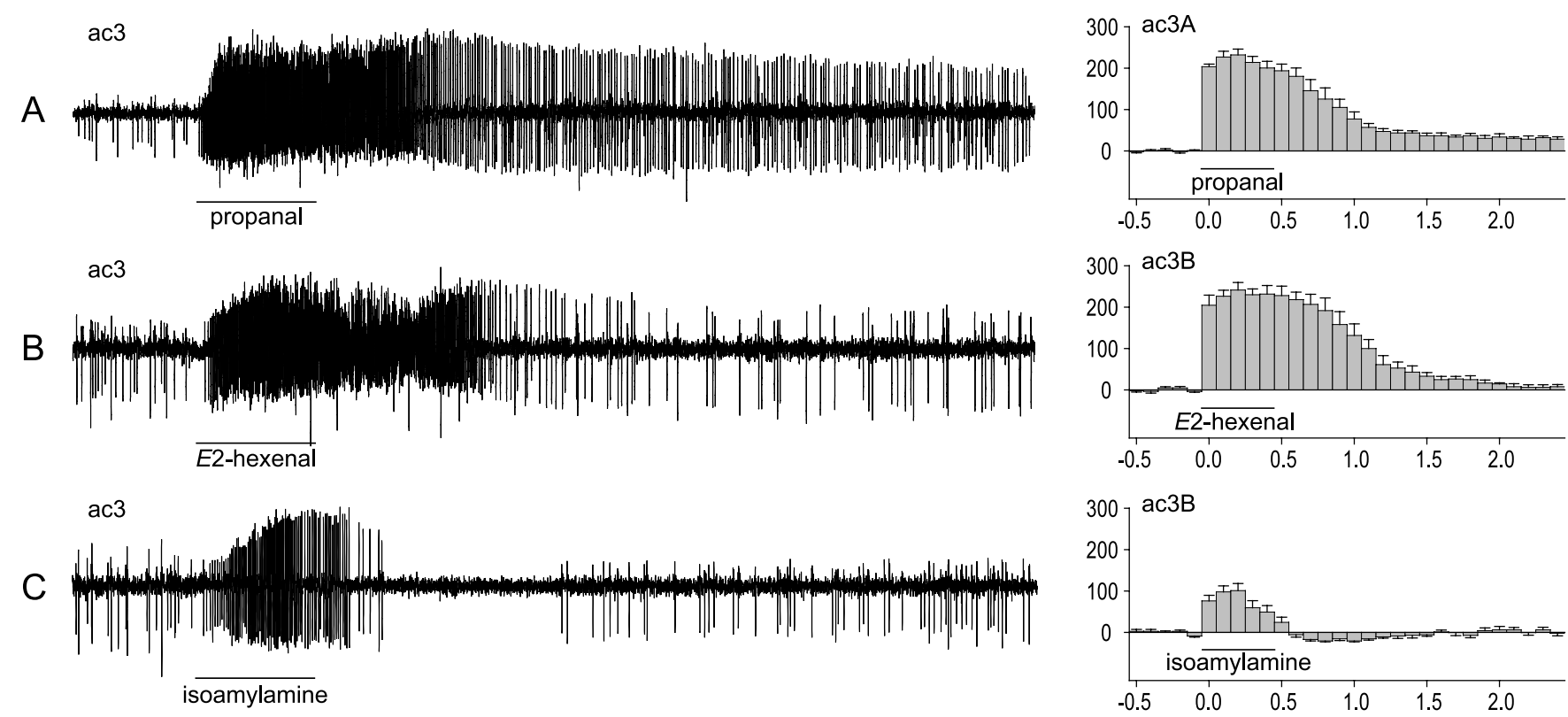

ac2
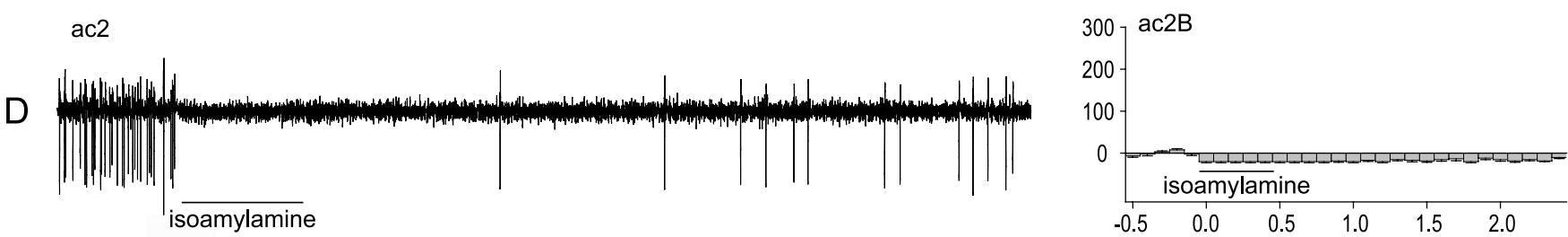

Figure 4. Differing temporal dynamics of coeloconic ORN responses. Bars represent $0.5 \mathrm{~s}$ pulses of the indicated odors. $\boldsymbol{A}$, Response of ac $3 \mathrm{~A}$ to propanal. The amplitude of the A neuron increases after odor stimulation (see Results); the smaller spikes of the $B$ cell can be seen in the second half of the trace. The vertical axis of the trace is reduced relative to the other traces in the figure. $B$, Response of ac3B to E2-hexenal. $C$, Response of ac 3 B to isoamylamine. The A cell is inhibited, as shown in Table 1. D, Inhibition of ac $2 B$ by isoamylamine. The A cell is also inhibited. In all graphs, $n=$ 6. Error bars indicate SEMs.

the onset of the odor stimulus, with the inhibition lasting throughout the entire recording period. During the final $100 \mathrm{~ms}$ period shown in the graph, the firing rate is $10.3 \pm 2.5$ spikes/s below the spontaneous firing frequency; after $10 \mathrm{~s}$, the rate was still $12 \pm 3.0$ spike/s $(n=6)$ below the spontaneous firing frequency.

\section{Humidity sensors}

Recordings from acl showed changes in spike frequency after changes in humidity. When the humidity was increased, the firing rate increased; when the humidity decreased, the firing rate decreased (Fig. 5A). Specifically, a change from dry air $(0 \% \mathrm{RH})$ to moist air $(\sim 100 \% \mathrm{RH})$ produced a rapid increase of $\sim 50$ spikes/s in the firing rate, and this increase was maintained until the humid air was again replaced by dry air, at which time the firing frequency fell abruptly (Fig. 5B, left two graphs). A similar response was observed in recordings from ac2 sensilla, although the change in firing frequency was smaller: $\sim 20$ spikes/s (Fig. $5 B$, right two graphs).

The changes in firing frequencies are sustained after a change in humidity. The graphs in Figure $5 B$ show that, in ac1, the changes in firing frequency are sustained during a $5 \mathrm{~s}$ recording period, i.e., the firing frequency is relatively stable during the course of this period. In a separate experiment, we recorded activity during a $10 \mathrm{~s}$ period after a sustained change in humidity. We found that, in the case of acl, the firing frequency was elevated by $47 \pm 7$ spikes/s after $5 \mathrm{~s}$ of increased humidity and by
$41 \pm 8$ spikes $/ \mathrm{s}$ after $10 \mathrm{~s}$ ( $n=6$ in both cases). The corresponding numbers for ac 2 were $25 \pm 5$ and $28 \pm 5$ spikes/s, respectively. Likewise, the decreased frequencies at $5 \mathrm{~s}$ and those at $10 \mathrm{~s}$ were similar after a decrease in humidity $(-33 \pm 6$ spikes/s at $5 \mathrm{~s}$ and $-40 \pm 8$ spikes/s at $10 \mathrm{~s}$ for ac1; $-23 \pm 5$ spikes/s at $5 \mathrm{~s}$ and $-18 \pm 5$ spikes/s at $10 \mathrm{~s}$ for ac2; $n=6$ in all cases).

We were initially motivated to test humidity because the ac1B and ac2B cells showed few, if any, excitatory responses to any of the diverse odorants we had tested (Table 1), and we therefore wished to test the hypothesis that they responded to humidity. The responses recorded from acl and ac2 after humidity changes are consistent with this hypothesis. However, we were unable to confirm with confidence that the humidity responses of acl and ac2 in fact derive from ac1B and ac2B cells: the spike amplitudes of $A$ and $B$ cells after changes in humidity were difficult to resolve. We cannot exclude the possibility of two humidity-responsive cells: one that responds to increases in humidity and one that responds to decreases, as has been found in the migratory locust (Altner et al., 1981) and in the cockroach (Tichy, 2003). Because experimentally induced changes in humidity can be accompanied by small changes in temperature, and because temperaturesensitive cells have been found in some cases to be combined with humidity-sensitive cells in coeloconic sensilla of other insects (Altner et al., 1977, 1981), we also tested ac1 and ac2 sensilla for responses to changes in temperature. Limited data revealed no responses to temperature changes in ac1 or ac 2 or in ac 3 or ac4.

We note that the presence of humidity had little, if any, effect 

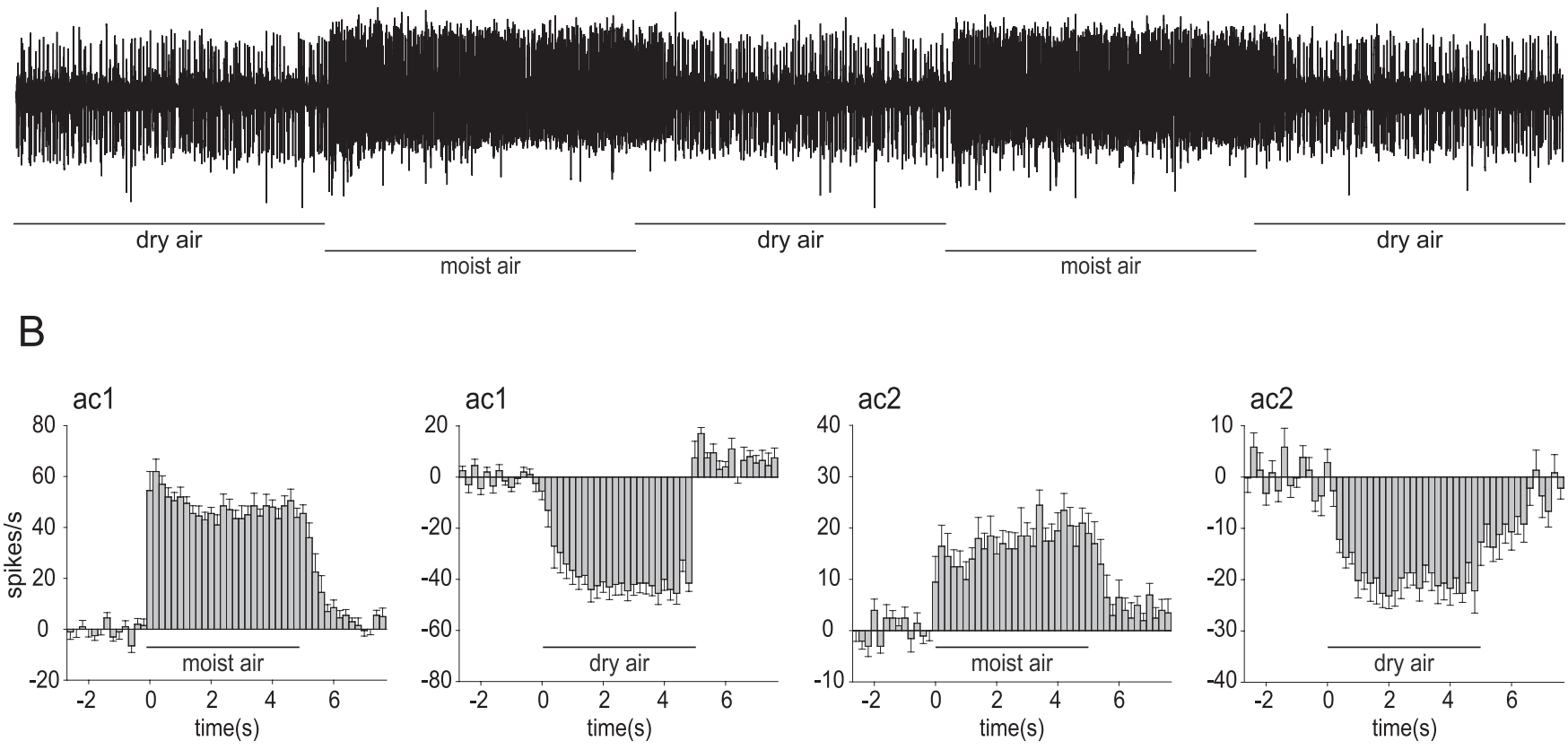

Figure 5. Responses to humidity. $\boldsymbol{A}$, Recording from an ac 1 sensillum. Bars represent $5 \mathrm{~s}$ pulses. $\boldsymbol{B}$, Firing frequency (bin width, $200 \mathrm{~ms}$ ) after changes in humidity. The two left panels show data from ac1 sensilla; the right panels represent ac2 sensilla. The vertical scales are different for the two sensillum types. In panels showing responses to "moist air," the fly was exposed to dry air for 5 min before switching to humid air for $5 \mathrm{~s}$, followed by a switch back to dry air. Likewise, in the panels showing responses to "dry air," the fly was exposed for 5 min to humid air before switching to dry air for $5 \mathrm{~s}$, followed by a switch back to humid air. In each graph, the number of spikes was counted during the $2.5 \mathrm{~s}$ period immediately before the change in humidity indicated by the bar. The average firing frequency during this $2.5 \mathrm{~s}$ prestimulus period was computed and subtracted from that of all bins in the graph. The spike counts represent all impulses recorded from each sensillum. $n=10$ sensilla in each graph. Error bars indicate SEMs.

on the ammonia response of ac1A or the 1,4-diaminobutane response of ac2A, in limited testing. A $500 \mathrm{~ms}$ pulse of ammonia increased the firing frequency of ac1A by $159 \pm 14$ spikes/s $(n=$ $24)$ in moist air and by $154 \pm 6$ spikes/s $(n=10)$ in dry air. Likewise, a $500 \mathrm{~ms}$ pulse of 1,4-diaminobutane increased the firing frequency of ac2A by $276 \pm 8$ spikes/s $(n=35)$ in moist air and $245 \pm 10$ spikes/s in dry air $(n=10)$.

\section{Genetic and molecular manipulation of Or35a eliminates odor response of ac3B}

Recently, the odor specificities of the Or family of antennal odor receptors were tested individually by expression of each receptor in an in vivo expression system (Hallem et al., 2004). Eleven of the tested receptors were found to confer odor response spectra that matched those of previously defined basiconic ORNs, and one, Or35a, conferred a response spectrum that corresponded to that of an undefined coeloconic ORN. This response spectrum appears similar to that defined here for ac $3 \mathrm{~B}$, but we wished to determine directly the identity of the Or35a-expressing cell and to investigate the physiological effects of eliminating Or35a in vivo.

We obtained a stock containing a transposable element insertion 283 bp upstream of the Or35a translation start codon (Fig. $6 A)$. The insertion lies in a region of noncoding DNA that is conserved between D. melanogaster and D. yakuba, suggesting that the region contains regulatory sequences essential for normal gene expression. Electrophysiological recordings from this insertion line revealed a population of coeloconic sensilla that gave responses characteristic of ac $3 \mathrm{~A}$ but not of ac3B (Fig. $6 \mathrm{~B}$, middle graph; compare with left graph). For example, the strong excitatory response to butanol recorded from ac3 sensilla in wild type (Fig. 6C, top trace) is not observed in these Or35a mutant sensilla (Fig. 6C, middle trace). We examined the mutant sensilla in detail with 30 odors and found that all contained two ORNs: one that exhibited an odor response spectrum consistent with ac3A and one ORN that did not respond to any of 30 tested odors, most of which elicit strong or moderate responses from wild-type ac3B cells. Among $\sim 30$ coeloconic sensilla analyzed, we found none in which an ac3A cell, identified by virtue of its strong response to propionic acid, was paired with an odor-responsive cell. All ac3A cells in this analysis were paired with a cell that yielded spontaneous impulses, albeit at reduced frequency $(6 \pm 2$ spikes/s compared with $26 \pm 2$ spikes/s in wild type for ac3B). Spikes in the insertion line did not show bursting, as was observed in cells suffering loss of the Or $22 a$ or Or $43 b$ genes (Dobritsa et al., 2003; Elmore et al., 2003).

We next generated an Or35a-GAL4 construct and used it to drive synthesis of the reaper (rpr) cell death gene. Recordings from coeloconic sensilla of Or35a-GAL4;UAS-rpr revealed a population of sensilla that contain apparently normal ac3A cells but no other cells (Fig. $6 B$, right graph, $C$, bottom trace). We observed no spikes other than those attributable to ac3A.

The simplest interpretation of these results is that Or35a is the receptor that confers the odor response of $\mathrm{ac} 3 \mathrm{~B}$ and that the function of ac3A does not depend on the presence of the neighboring ac3B cell.

\section{Discussion}

The first step in the coding of an olfactory stimulus is the activation of ORNs in olfactory organs. In this study, we examined in detail the coding of olfactory information by ORNs in a major 
A

B
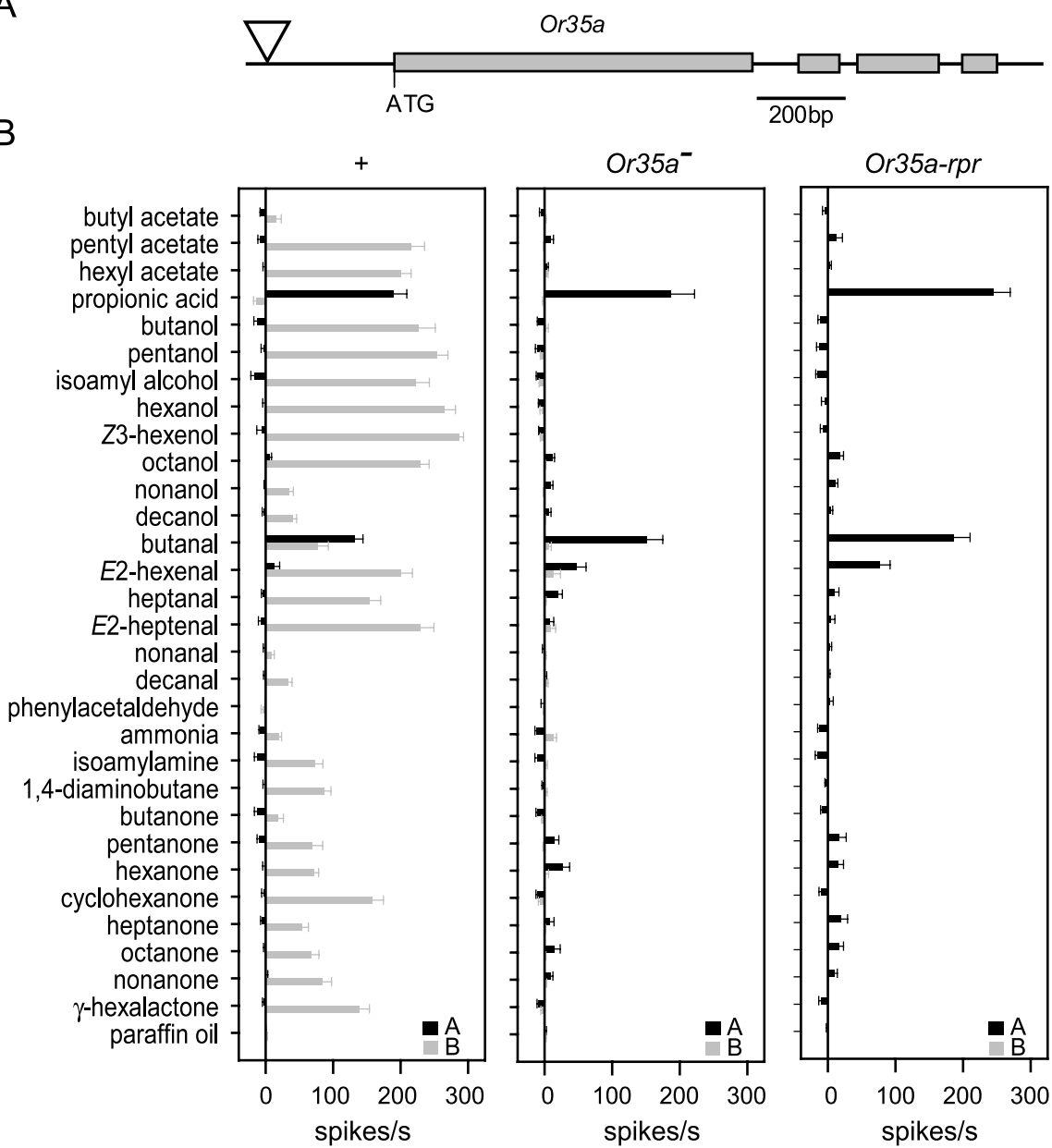

C
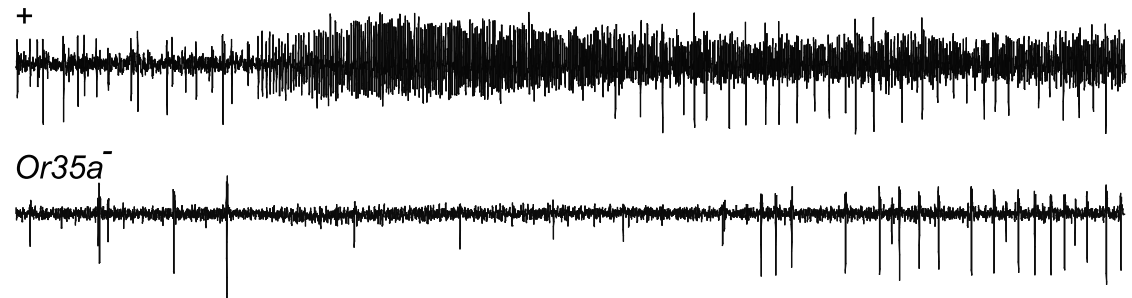

Or35a-rpr

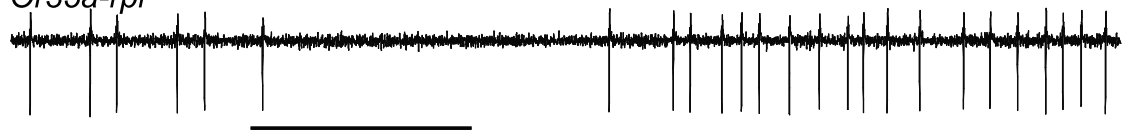

butanol

Figure 6. Or $35 a$ and the ac 3 sensillum. $\boldsymbol{A}$, The Or $35 a$ locus, showing insertion site (triangle) of a transposable element. $\boldsymbol{B}$, Responses of wild type (left), an Or35a insertion mutant (center), and an Or35a-GAL4;UAS-rpr line (right). The Or35a insertion mutant was homozygous for the insertion, and Or35a-GAL4;UAS-rpr was homozygous for both the Or35a-GAL4 driver and the UAS-rpr element. The data in the left panel are taken from Figure 3, except in the case of propionic acid, which was used in this experiment at a $10^{-1}$ dilution and for which $n=27$. Middle panel, $n=8$ for all odorants; right panel, $n=11$ for all odorants. $C$, Traces of the different genotypes. The bar represents a 0.5 s pulse. Error bars indicate SEMs.

class of olfactory sensilla, the coeloconic sensilla, whose function in Drosophila has until now been essentially unexplored.

Physiological recordings identified four distinct functional types of coeloconic sensilla on the antennal surface. Each type contains at least two ORNs, and the data show that there are at least seven distinguishable classes of ORNs; we were unable to distinguish with confidence the activities of the different ORNs in ac4 sensilla, but it is clear from the response to phenylacetaldehyde, for example, that at least one of the ORNs in ac4 is distinct from those in ac1, ac2, and ac3. We note that an anatomical study of coeloconic sensilla revealed two subtypes: one containing two neurons and the other containing three neurons (Shanbhag et al., 1999). It thus seems likely that at least one functional type, such as ac4, contains three ORNs.

We identified neurons in the Drosophila antenna that respond strongly to humidity. Hygroreception is critical in the insect world. Many insects, such as mosquitoes, lay eggs in water, and dry environments can lead to desiccation and death. Drosophila exhibits preferences when given a choice between environments of different humidities (Sayeed and Benzer, 1996); however, the molecular and genetic basis of hygroreception is not understood, and even its cellular basis has until now been unknown in Drosophila.

In defining a cellular basis of hygroreception in Drosophila, we provided a focus for a molecular and genetic analysis of the mechanism of transduction. We note that ablation experiments had provided evidence in a previous study that hygroreception localizes to the arista, a feathery projection extending from the third antennal segment (Sayeed and Benzer, 1996). However, this study also found that spineless ${ }^{\text {aristapedia }}$, a homeotic mutant in which aristae are transformed into leg-like structures, had normal hygroreception. Our studies do not exclude the possibility of hygroreceptive cells associated with the arista or elsewhere; however, our identification of hygroreceptors in coeloconic sensilla is consistent with studies of other insects in which hygroreceptive cells were identified in morphologically similar sensilla (Altner et al., 1977; van den Broek and Den Otter, 2000).

The temporal dynamics of humidity responses, shown in the graphs of Figure 5 , appear largely tonic. The responses to odors vary in their dynamics (Fig. 4). The diverse temporal dynamics shown by coeloconic ORNs may reflect another degree of freedom used by the antenna in odor coding: the temporal structure of olfactory information has been shown to be critical in odor coding in a variety of systems (Laurent et al., 2001).

We note with interest that, although the transduction mechanisms underlying hygroreception and olfaction may differ, they are likely to be housed in a common cell (i.e., in a multimodal neuron: isoamylamine inhibits all neuronal activity in ac2 sensilla) (Figs. $2 D, 4 D$ ), including that of humidity-sensitive cells, suggesting that hygroreceptive cells contain a receptor for isoamylamine. 
We also identified amine detectors. One class of ORN, ac1A, is highly sensitive to ammonia, and another, ac2A, responds strongly to 1,4-diaminobutane. A particularly important example of amine detection in insects is the attraction of mosquitoes to ammonia, a component of human sweat (Braks et al., 2001). Ammonia-sensitive cells have been detected in double-walled, grooved-peg sensilla on the antenna of Anopheles mosquitoes (van den Broek and Den Otter, 2000) and Triatoma infestans, a vector of trypanosomiasis, or Chagas disease (Taneja and Guerin, 1997). Identification of these cells in Drosophila lays a foundation for examining the molecular genetics of amine detection, which, in turn, could be useful in designing new means of pest control.

The response spectra of the coeloconic ORNs vary remarkably in their apparent breadth of tuning. At one extreme is ac3B, which reveals exceptionally broad tuning. ac $3 \mathrm{~B}$ is excited by 36 of 45 odors at the test concentrations; there is evidence that some coeloconic sensilla in the moth Bombyx mori yield responses to a variety of acids and alcohols (Pophof, 1997). ac1B, in contrast, is excited by none of these 45 odors; it may be the cell in acl that responds to humidity. ac1A responds strongly to ammonia and weakly to two other amines, but shows no responses to the other 42 tested odors. The profile of ac2A suggests that it may have evolved to signal the presence of 1,4-diaminobutane. No strong excitatory responses were detected among the neurons of ac4, other than a modest response to phenylacetaldehyde, and it seems likely that they detect the presence of biologically significant stimuli that are not included in our odor set. Conclusions about the breadth of ORN tuning are limited by the number of odors tested. Odor space is vast and discontinuous, and the sampling of odors here is necessarily limited. Nonetheless, the pattern that emerges from this analysis is one of a single broadly tuned ORN, ac3B, and others that are excited by only one or a small number of stimuli.

In light of the widely varying tuning breadths of these ORNs, it seems plausible that the coeloconic sensilla have evolved in large part to signal the presence of a small number of specific chemosensory stimuli, such as water vapor, ammonia, and 1,4diaminobutane, also known as putrescine. The exceptionally broad spectrum of ac $3 \mathrm{~B}$ could have evolved as a more general sensor to signal the simultaneous presence of food sources. The coeloconic sensilla have an ancient origin (Steinbrecht, 1997), and perhaps their specificities reflect the most basic needs of an ancestral insect.

The specificities of these neurons are also of interest in light of the recent construction of a receptor-to-neuron map for the Drosophila antenna (Dobritsa et al., 2003; Hallem et al., 2004). Or genes were individually expressed in a mutant in which a particular ORN, ab3A, loses its odorant response because of a deletion that removes two odorant receptor genes, Or22a and Or22b. When particular Or genes were expressed in this mutant ORN, they conferred in many cases an odor response spectrum that matched that of a defined ORN. In this way, individual Or genes could be assigned to particular ORNs. Testing of virtually the entire repertoire of antennal Or genes mapped many receptor genes to basiconic ORNs, but only one, Or35a, to a coeloconic ORN. Because a number of receptors in that study conferred response profiles that did not match those of any defined ORNs, we further tested these unmapped receptors, using a panel of odorants from Table 1 that the present study has identified as diagnostic for particular coeloconic ORN classes. However, we were again unable to map any of these receptors to coeloconic ORNs (data not shown).

Why has only a single class of coeloconic ORN been found to derive its odor response profile from an Or gene? One possible interpretation is that some coeloconic ORNs, for example those sensitive to humidity or ammonia, do not rely on receptors of the Or family to detect the molecules that activate them. We note that no Or gene was mapped to ab1C, a basiconic ORN that is a sensor of $\mathrm{CO}_{2}$. A $\mathrm{Gr}$ (Gustatory receptor) gene (Clyne et al., 2000) has been mapped to this neuron (Suh et al., 2004), although at present there is no evidence that it is a $\mathrm{CO}_{2}$ receptor. In the present study, we showed that Or35a is necessary for the response of ac3B. An important goal now is to determine which receptors are necessary for the responses of the other coeloconic neurons. The analysis of coeloconic ORNs presented here provides a foundation for investigating the molecular and genetic basis of the mechanisms by which coeloconic ORNs transduce chemosensory signals. It also provides a basis for investigating the roles of these neurons in olfactory-driven behaviors.

\section{References}

Altner H, Sass H, Altner I (1977) Relationship between structure and function of antennal chemo-, hygro-, and thermoreceptive sensilla in Periplaneta americana. Cell Tissue Res 176:389-405.

Altner H, Routil C, Loftus R (1981) The structure of bimodal chemo-, thermo-, and hygroreceptive sensilla on the antenna of Locusta migratoria. Cell Tissue Res 215:289-308.

Braks MAH, Meijerink J, Takken W (2001) The response of the malaria mosquito, Anopheles gambiae, to two components of human sweat, ammonia and L-lactic acid, in an olfactometer. Physiol Entomol 26:142-148.

Clyne P, Grant A, O'Connell R, Carlson JR (1997) Odorant response of individual sensilla on the Drosophila antenna. Invert Neurosci 3:127-135.

Clyne PJ, Warr CG, Carlson JR (2000) Candidate taste receptors in Drosophila. Science 287:1830-1834.

de Bruyne M, Clyne PJ, Carlson JR (1999) Odor coding in a model olfactory organ: the Drosophila maxillary palp. J Neurosci 19:4520-4532.

de Bruyne M, Foster K, Carlson JR (2001) Odor coding in the Drosophila antenna. Neuron 30:537-552.

Dobritsa AA, Van der Goes van Naters WM, Warr CG, Steinbrecht RA, Carlson JR (2003) Integrating the molecular and cellular basis of odor coding in the Drosophila antenna. Neuron 37:827-841.

Elmore T, Ignell R, Carlson JR, Smith DP (2003) Targeted mutation of a Drosophila odor receptor defines receptor requirement in a novel class of sensillum. J Neurosci 23:9906-9912.

Goldman A, van der Goes van Naters W, Warr C, Lessing D, Carlson JR (2005) Coexpression of two functional odor receptors in one neuron. Neuron 45:661-666.

Guillet J, Bernard J (1972) Shape and amplitude of the spikes induced by natural or electrical stimulation in insect receptors. J Insect Physiol 18:2155-2171.

Haggart D, Davis E (1980) Ammonia-sensitive neurons on the first tarsi of the tick, Rhipicephalus sanguineus. J Insect Physiol 26:517-523.

Hallem EA, Carlson JR (2004) The odor coding system of Drosophila. Trends Genet 20:453-459.

Hallem EA, Ho M, Carlson JR (2004) The molecular basis of odor coding in the Drosophila antenna. Cell 117:965-980.

Hildebrand JG, Shepherd GM (1997) Mechanisms of olfactory discrimination: converging evidence for common principles across phyla. Annu Rev Neurosci 20:595-631.

Kaissling K-E, Thorson J (1980) Insect olfactory sensilla: structure, chemical and electrical aspects of the functional organisation. In: Receptors for transmitters, hormones and pheromones in insects (Sattelle D, Hall LM, Hildebrand J, eds), pp 261-282. Amsterdam: Elsevier.

Kreher SA, Kwon JY, Carlson JR (2005) The molecular basis of odor coding in the Drosophila larva. Neuron 46:445-456.

Laurent G, Stopfer M, Friedrich RW, Rabinovich MI, Volkovskii A, Abarbanel HD (2001) Odor encoding as an active, dynamical process: experiments, computation, and theory. Annu Rev Neurosci 24:263-297.

Minor AV, Kaissling KE (2003) Cell responses to single pheromone molecules may reflect the activation kinetics of olfactory receptor molecules. J Comp Physiol A Neuroethol Sens Neural Behav Physiol 189:221-230.

Park SK, Shanbhag SR, Dubin AE, de Bruyne M, Wang Q, Yu P, Shimoni N, D'Mello S, Carlson JR, Harris GL, Steinbrecht RA, Pikielny CW 
(2002) Inactivation of olfactory sensilla of a single morphological type differentially affects the response of Drosophila to odors. J Neurobiol 51:248-260.

Pophof B (1997) Olfactory responses recorded from sensilla coeloconica of the silkmoth Bombyx mori. Physiol Entomol 22:239-248.

Redkozubov A (1995) High electrical resistance of the bombykol cell in an olfactory sensillum of Bombyx mori: voltage- and current-clamp analysis. J Insect Physiol 41:451-455.

Rumbo E (1989) The shape of extracellularly recorded nerve impulses. Chem Senses 14:361-369.

Sayeed O, Benzer S (1996) Behavioral genetics of thermosensation and hygrosensation in Drosophila. Proc Natl Acad Sci USA 93:6079-6084.

Shanbhag S, Muller B, Steinbrecht A (1999) Atlas of olfactory organs of Drosophila melanogaster. 1. Types, external organization, innervation and distribution of olfactory sensilla. Int J Insect Morphol Embryol 28:377-397.

Steinbrecht A (1997) Pore structures in insect olfactory sensilla: a review of data and concepts. Int J Insect Morphol Embryol 26:29-245.

Stensmyr MC, Giordano E, Balloi A, Angioy AM, Hansson BS (2003) Novel natural ligands for Drosophila olfactory receptor neurones. J Exp Biol 206:715-724.

Suh GS, Wong AM, Hergarden AC, Wang JW, Simon AF, Benzer S, Axel R, Anderson DJ (2004) A single population of olfactory sensory neurons mediates an innate avoidance behaviour in Drosophila. Nature 431:854-859.

Taneja J, Guerin P (1997) Ammonia attracts the haematophagous bug Triatoma infestans: behavioural and neurophysiological data on nymphs. J Comp Physiol A Neuroethol Sens Neural Behav Physiol 181:21-34.

Tichy H (2003) Low rates of change enhance effect of humidity on the activity of insect hygroreceptors. J Comp Physiol A Neuroethol Sens Neural Behav Physiol 189:175-179.

TNO (2004) Volatile compounds in food. Qualitative and quantitative data (www.voeding.tno.nl/vcf).

van den Broek I, Den Otter C (2000) Odour sensitivity of antennal olfactory cells underlying grooved pegs of Anopheles gambiae s.s. and An. quadriannulatus. Entomol Exp Appl 96:167-175. 